\title{
Pressure-Driven Filling of Closed-End Microchannel: Realization of Comb-Shaped Transducers for Acoustofluidics
}

\author{
Wei Guo, ${ }^{1, \S}$ Adrian J. T. Teo, ${ }^{2, \S}$ Alfonso M. Gañán-Calvo, ${ }^{3,}{ }^{*}$ Chaolong Song, ${ }^{4}$ Nam-Trung Nguyen, ${ }^{2}$ \\ Heng-Dong Xi, ${ }^{1, \dagger}$ and Say Hwa Tan ${ }^{2, \ddagger}$ \\ ${ }^{1}$ School of Aeronautics, Northwestern Polytechnical University, 127 West Youyi Rd, Xi'an, Shaanxi, China \\ ${ }^{2}$ Queensland Micro and Nanotechnology Centre, Griffith University, 170 Kessels Road, Queensland 4111, \\ Australia \\ ${ }^{3}$ Departamento de Ingeniería Aeroespacial y Mecánica de Fluidos, Universidad de Sevilla, E-41092 Sevilla, Spain \\ ${ }^{4}$ School of Mechanical Engineering and Electronic Information, China University of Geosciences, 388 Lumo Rd, \\ Wuhan, Hubei, China
}

(Received 8 April 2018; revised manuscript received 2 August 2018; published 20 November 2018)

\begin{abstract}
We demonstrate the complete filling of both deionized water (DI water) and liquid metal (eutectic gallium-indium, EGaIn) into closed-end microchannels driven by a constant pressure at the inlet. A mathematical model based on gas diffusion through a porous polydimethylsiloxane (PDMS) wall is developed to unveil the physical mechanism in the filling process. The proposed theoretical analysis based on our model agrees well with the experimental observations. We also successfully generate traveling surface acoustic waves by actuating interdigitated microchannels filled with EGaIn. Our work provides significant insights into the fabrication of liquid electrodes that can be used for various acustofluidics applications.
\end{abstract}

DOI: 10.1103/PhysRevApplied.10.054045

\section{INTRODUCTION}

Acoustofluidics is the exquisite marriage between acoustics and microfluidics. The combination of both fields leverages inherent in situ advantages at the microscale [1-3]. Both acoustics and microfluidics address several shortcomings at the microscale, which can be extremely desirable in many chemical and biological applications. On the one hand, acoustics provides a noninvasive and noncontact mode of manipulation through propagating sound waves [4,5]. On the other hand, microfluidics provides a miniaturized platform to perform different micro total analysis (MicroTAS) systems with significant reductions in the speed of reactions and the amount of reagents [6]. In recent years, this integration has been gaining much attention, evident with a themed collection of tutorials in Lab on a Chip [7]. A broad range of articles discuss various issues from basic governing equations to different applications. This collection ascertains both the popularity and importance of acoustofluidics.

Surface acoustic waves (SAW), a sub-branch of acoustics pioneered by White and Voltmer [8], have been extensively used in electronic circuits [9]. To date, numerous applications of SAW have been developed to perform

\footnotetext{
*amgc@us.es

†hengdongxi@nwpu.edu.cn

†sayhwa.tan@griffith.edu.au

$\S$ These authors contributed equally to this work.
}

tasks in droplet-based microfluidics. These tasks include operations such as droplet generation [10,11], coalescence [12], and sorting [13]. In these applications, SAWs are commonly generated using comb-shaped transducers [14]. These interdigitated transducers (IDTs) are fabricated using traditional photolithography and lift-off techniques [15] that are often expensive, laborious, and time consuming. Often, the transducers and fluidic channels are fabricated separately and have to be painfully aligned during the integration. Misalignment results in inappropriate wave propagation [16] at unsuitable locations. In order to circumvent the complex fabrication procedures, we recently demonstrated that microsolidics can be used to fabricate self-aligned IDTs [17] for acoustofluidics. Microsolidics, a technique introduced by Whitesides [18], has been extensively used to fabricate various types of electrodes [19,20] and electromagnets in polydimethylsiloxane (PDMS). Recently, Nam et al. used a modified microsolidics technique to fabricate serpentine-shaped electrodes for acoustofluidics [21]. In the work, eutectic gallium-indium (EGaIn), a conductive liquid metal at room temperature, is used to pervade the microchannels to induce the required alternating electric fields. This openend channel design mimics the double-electrode IDTs, which are not common in microfluidic applications. The double-electrode IDTs (serpentine-shaped electrodes) differ from conventional comb-shaped IDTs and have several drawbacks. For example, a nonactive region [22] above the 
interdigitated fingers wastes space. The serpentine design also limits the number of electrode pairs due to the space constraint in a microfluidic device. The number of electrode pairs directly affects the strength of the resulting SAWs [23]. In designing complex electrode structures such as the focused interdigitated transducers (FIDTs) [24] or slanted interdigitated transducers (SIDTs) [25], the modification of the serpentine design to the FIDTs/SIDTs geometries is very tedious and time consuming. In a physical aspect, the serpentine-shaped electrodes generate SAWs at resonance frequencies that are lower than conventional single-electrode IDTs [21]. The lower resonance frequencies induce lower streaming velocities [26] and weaker acoustic forces within the microsystems [27].

To overcome the above limitations, self-aligned combshaped single-electrode IDTs may be preferred and better received by the microfluidics community. In contrast to the popular belief that the filling of closed-end microchannels may be time consuming and tedious [21], we show in this work that both deionized (DI) water and liquid metal (EGaIn) can completely fill up the microchannels within minutes using a pressurized source. This demonstration exploits the distinctive porous nature of PDMS. We also provide the detailed experimental and theoretical explanations on the filling process of EGaIn in closed-end combshaped microchannels. To the best of our knowledge, no literature can be found to characterize and model the complete filling process of DI water/EGaIn in microchannels driven by a constant pressure at the inlet. We develop a theoretical model by considering the gas diffusion through the porous PDMS. The analytical results of the model show good agreement with the experimental data. As a proof of concept, we also show that the proposed comb-shaped single-electrode IDTs can be used to generate SAWs to induce streaming in an open-space droplet, demonstrating that our proposed technique is both feasible and practical in nature.

\section{EXPERIMENTAL SETUP}

Figure 1 shows the schematic of the experimental setup. The PDMS microchannels are fabricated using standard photo- and soft-lithography [28]. The channel width $w=$ $200 \mu \mathrm{m}$, height $h \sim 47 \mu \mathrm{m}$, and length $L=2750 \mu \mathrm{m}$. The width and the height of the main (root) channel are about 420 and $47 \mu \mathrm{m}$, respectively. A pressure controller [29] (Elveflow, OB1 MK3) is connected to one end of the channel with the other end closed. Both DI water and EGaIn are filled into the channels under different constant pressures $P_{i}\left(P_{i}=200,400,800,1200 \mathrm{mbar}\right.$, respectively). We first evaluate the flow characteristics of DI water in a closedend channel to form the basic theoretical model. EGaIn is then filled into the channels for comparison and evaluation using the same model. The DI water is mixed with a small amount of food dye (blue) for visualization and data evaluation. The addition of the food dye does not change the flow properties of the DI water. The PDMS device is mounted on an inverted microscope (Nikon TiE, Japan), while images are captured using a monochrome camera (Miro 3, Vision Research) at a frame rate of 10 frames/s to capture the entire filling process. In the case of DI water, the shortest filling time achieved is less than 6 min. Interestingly, for the case of EGaIn, the shortest filling time achieved is less than 2 min with the same device design. Here, we would like to stress that the filling time mentioned above corresponds to the filling time for nine adjacent fingers. Theoretically, the filling time does not depend on the number of channels involved as long as they are parallel and have the same common root channel. We observed experimentally that all of the comb-shaped fingers connected to the same root channel are found to be completely filled almost at the same time. Negligible differences are observed between each finger. Based on the experimental data, we assume that the pressure imposed in the liquid is the same as the inlet pressure.

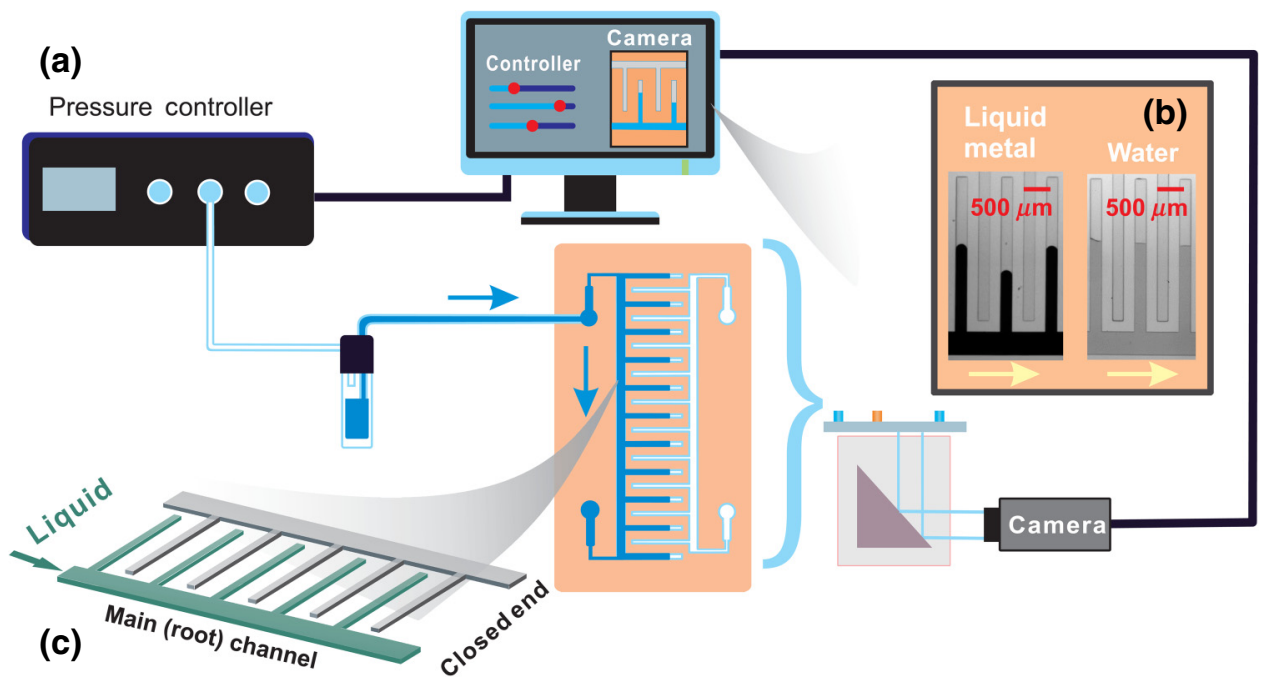

FIG. 1. Experimental setup: (a) Pressure control system. (b) Images obtained from the camera. (c) $3 \mathrm{D}$ schematic of the PDMS channels. 


\section{THEORETICAL MODEL AND EXPERIMENTAL RESULTS}

The filling process of the closed-end microchannels shown in Fig. 1 is investigated. Assuming that a constant driving pressure is imposed at the inlet, the advancement of the filling liquid is accompanied by the escape of air from the closed channel. In this case, air escapes by diffusion through the porous PDMS walls. This process is different from the classical capillary filling model, where capillary force and viscous force are predominant [30]. The rationale is based on the fact that the diffusion time of momentum $\left(t_{v}=h w / v=9.4 \mathrm{~ms}\right.$, where $v$ is kinetic viscosity coefficient of the liquid) is negligible compared to that of the diffusion of air into PDMS or water $\left(t_{D}=h w / D_{\text {gas }}=4.7 \mathrm{~s}\right.$, where $D_{\text {gas }}$ is the diffusion coefficient of air into PDMS or water, and $D_{\text {gas }} \sim 4.5 \times 10^{-9} \mathrm{~m}^{2} \mathrm{~s}^{-1}$ [31]). Given that the volume of PDMS is immense compared to that of the liquid, only a tiny fraction of air diffuses into the liquid. Thus, the same pressure is imposed in the liquid everywhere and is equal to the applied pressure from the controller. Furthermore, the system is considered to be isothermal. We neglect liquid vapor and condensation drops on the channel wall. We propose here a dimensionless number $G$ that can be used to characterize the relative weight of viscous relaxation time along the channel for a given pressure to the time of air diffusion into PDMS, $G=$ $12 \mu D_{\text {gas }} L^{2} /\left[w h^{3}\left(P_{0}+P_{i}\right)\right]$, where $P_{0}$ is the atmosphere pressure. Our experiment here has $G \sim 10^{-4}$, meaning that the gas diffusion dominates the viscous effects. This situation is different from the case of capillary filling in a closed-end nanochannel with a nonpermable wall, where $G \sim 10^{6}$ and force analysis is necessary [30]. On the other hand, the channel height $h$ and the channel width $w$ are much larger than those of nanochannels; hence, the resisting surface tension pressure in the filling process of DI water is relatively small compared to the imposed pressure. The Reynolds number $\left(\operatorname{Re}=w U / v \sim 10^{-3}\right.$, where $U$ is the velocity of the liquid, e.g., the DI water, in the finger) is relatively small in our experiments. Hence, inertial forces of the filling liquid can be neglected. In the filling process with DI water, the trapped air pressure is essentially equal to that of the filling liquid, $P_{i}+P_{0}$.

For mathematical simplicity, we can model our microchannels with a rectangular cross section as the equivalent cylindrical channel with the same perimeter, which gives $2(h+w)=\pi D$ or $D=2(h+w) / \pi$, where $D$ is the diameter of the equivalent cylindrical channel, as depicted in Fig. 2(a). With this equivalence, we can model the system in polar coordinates and avoid redundant calculations in Cartesian coordinates. Next, we consider the dynamic behavior of the trapped air both in the channel and in the PDMS domain shown in Fig. 2(b). Let $P(r)$, $Y(r)$, and $C(r)$ denote the pressure of air in the PDMS domain, mass, and volume fraction of gas in the PDMS
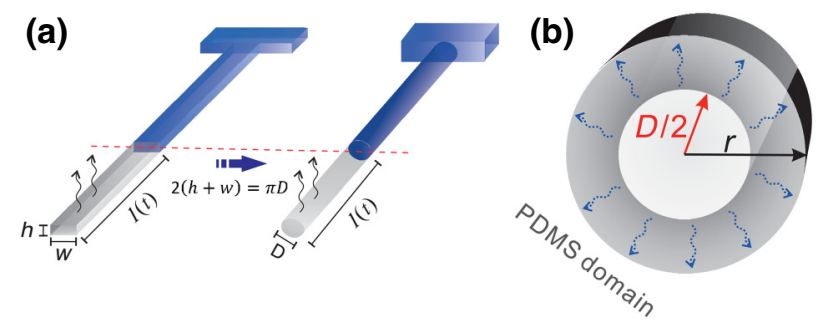

FIG. 2. (a) Model equivalence from the microchannel with rectangular cross section to that with circular cross section. The equivalence is based on the same perimeters of the two different geometries but they do not differ in channel length. (b) Calculation domain in the model. The shadow area is the PDMS domain in polar coordinates and the hollow area is the channel filled with trapped air under the pressure of $P_{0}+P_{i}$.

domain, respectively. According to the study of Sadrzadhel et al. [32], nitrogen and oxygen have similar permeability: $\partial C_{\mathrm{N}_{2}} / \partial P=9.35 \times 10^{-7} \mathrm{~Pa}^{-1}$ and $\partial C_{\mathrm{O}_{2}} / \partial P=$ $1.87 \times 10^{-6} \mathrm{~Pa}^{-1}$ in PDMS and, thus, we have

$$
\left\{\begin{array}{l}
\frac{\partial Y_{\mathrm{N}_{2}}}{\partial P}=\frac{\rho_{\mathrm{N}_{2}}}{\rho_{\mathrm{PDMS}}} \frac{\partial C_{\mathrm{N}_{2}}}{\partial P}=1.1 \times 10^{-9} \mathrm{~Pa}^{-1}, \\
\frac{\partial Y_{\mathrm{O}_{2}}}{\partial P}=\frac{\rho_{\mathrm{O}_{2}}}{\rho_{\mathrm{PDMS}}} \frac{\partial C_{\mathrm{O}_{2}}}{\partial P}=2.5 \times 10^{-9} \mathrm{~Pa}^{-1} .
\end{array}\right.
$$

Hence, we can calculate the mass fraction of gas in the PDMS domain under a given pressure $P$ as $Y=\partial Y / \partial P \times$ $P$, which governs the boundary conditions for the liquid filling process.

We define nondimensional variables $\eta=2 r / D$ and $\tau=$ $t / t_{0}$, where $t_{0}=D^{2} /\left(4 \times D_{\text {gas }}\right)=(h+w)^{2} /\left(\pi^{2} D_{\text {gas }}\right)$ denotes the characteristic diffusion time, and $D_{\text {gas }} \sim 4.5 \times$ $10^{-9} \mathrm{~m}^{2} \mathrm{~s}^{-1}$ is the diffusion coefficient of both $\mathrm{N}_{2}$ and $\mathrm{O}_{2}$ in PDMS (almost the same). For each individual finger, the filling time $t$ starts from the moment that the liquid enters the finger. The diffusion of gas into PDMS is governed by Fick's second law and can be rewritten as a nondimensional equation:

$$
\frac{\partial Y}{\partial \tau}=\frac{1}{\eta} \frac{\partial Y}{\partial \eta}+\frac{\partial^{2} Y}{\partial \eta^{2}} .
$$

Equation (2) is a partial differential equation with two variables. We introduce a self-similar variable $\xi=\eta^{2} /(4 \tau)$ and the partial differential equation can be transformed into an ordinary differential equation:

$$
\xi Y^{\prime \prime}+(1+\xi) Y^{\prime}=0
$$

with the boundary conditions

$$
\left\{\begin{array}{l}
Y\left(\xi_{0}\right)=Y_{0} \equiv\left(1+P_{i} / P_{0}\right) Y_{\infty} \\
Y(\xi \rightarrow \infty)=Y_{\infty}
\end{array}\right.
$$


where $\xi_{0}$ reflects the initial situation of the PDMS already loaded with air at atmospheric pressure $P_{0}$ before the liquid enters the channel. In this regard, it should have the same value for all different pressures and experiments performed with the same device. In our case, $\xi_{0}$ is fixed when DI water is injected at different pressures. As for the differences in the characteristic features of the geometries used in the model and the actual device, $\xi_{0}$ would be used as the fitting parameter for the model of each configuration.

Equation (3) has the analytical solution:

$$
Y(\xi)=Y_{\infty}\left[1+\frac{Y_{0}-Y_{\infty}}{Y_{\infty}} \frac{\operatorname{Ei}(-\xi)}{\operatorname{Ei}\left(-\xi_{0}\right)}\right],
$$

where $\operatorname{Ei}(x)$ is the exponential integral function and expressed as $\operatorname{Ei}(x)=-\int_{-x}^{\infty}\left(e^{-t} / t\right) d t$. Finally, to compute the diffusion velocity of air at the inner surface of the channel $v_{d}=-\left.D_{\text {gas }}(\partial Y / \partial r)\right|_{s}=-\left.D_{\text {gas }}(\partial Y / \partial r)\right|_{r=D / 2}$, one has

$$
v_{d}=-D_{\text {gas }} \frac{2 \pi}{h+w} \frac{\exp \left(-\frac{1}{4 \tau}\right)}{\operatorname{Ei}\left(-\xi_{0}\right)}\left(Y_{0}-Y_{\infty}\right) .
$$

Now, the advance of the liquid into the channel is governed by the following equation:

$$
\begin{aligned}
& \rho_{a} \frac{d l}{d t} h w+\left(0.79 \rho_{\mathrm{N}_{2}} v_{d, \mathrm{~N}_{2}}+0.21 \rho_{\mathrm{O}_{2}} v_{d, \mathrm{O}_{2}}\right) \times 2(h+w) l \\
& \quad=0
\end{aligned}
$$

with the initial condition of $l(0)=L$, where $l$ is the length of trapped air in the channel; $\rho_{a}=0.79 \rho_{\mathrm{N}_{2}}+0.21 \rho_{\mathrm{O}_{2}}$ is the density of air; and $v_{d}=-\left.D_{\text {gas }}(\partial Y / \partial r)\right|_{s}$ is the mass flux of the gas by Fick's first law, where $\partial Y /\left.\partial r\right|_{s}$ is the surface gradient of the mass gas fraction $Y$ in the PDMS (mass of gas per mass of PDMS plus gas). For simplicity, we assume that $v_{d}$ is the average value of the diffusion velocity along the whole inner surface of the channel $2(h+w) \times l$. Thus, based on Eq. (5), the solution for Eq. (6) is

$$
l(t)=L \times \exp \left[\frac{t}{t_{1}} \exp \left(-\frac{t_{0}}{4 t}\right)-\frac{t_{0}}{4 t_{1}} \Gamma\left(0, \frac{t_{0}}{4 t}\right)\right],
$$

where $\Gamma(0, x)$ denotes the Gamma function of the variable $x$, and

$$
t_{1}=\frac{\operatorname{Ei}\left(-\xi_{0}\right) h w \rho_{a} \times\left(P_{0} / P_{i}\right)}{4 \pi D_{\text {gas }}\left(0.79 \rho_{\mathrm{N}_{2}} Y_{\infty, \mathrm{N}_{2}}+0.21 \rho_{\mathrm{O}_{2}} Y_{\infty, \mathrm{O}_{2}}\right)} .
$$

Figure 3(a) shows the results of Eq. (7) in terms of dimensionless filling length $L_{f} / L$, where $L_{f}(t)=L-l(t)$ is the filling length of the liquid in microchannels. Here, we treat $k_{s}=-4 \pi / \operatorname{Ei}\left(-\xi_{0}\right)$ as a fitting factor for the experimental data, resulting in $\xi_{0}=2.103$. We can see a good agreement between the experimental data of DI water and the model.

Note that there is only one adjustable parameter in Eq. (7), $\xi_{0}$, and $P_{i}$ is an operational parameter. Again, consider that $\xi_{0}$ is a fixed fitting factor that reflects the initial
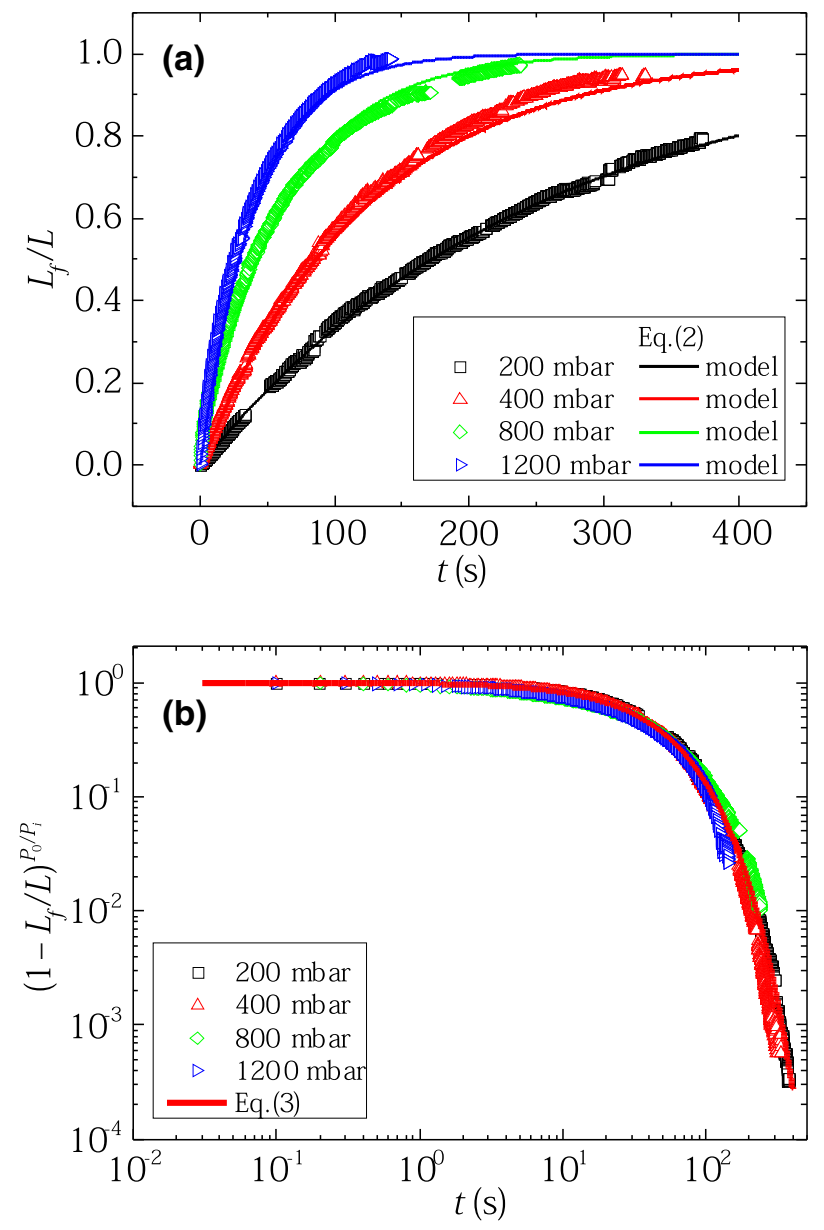

FIG. 3. Normalized filling length of DI water as a function of time at different inlet pressures. The dots are experimental data obtained from the videos using Matlab image processing. The solid lines are theoretical predictions from Eqs. (2) and (3), respectively.

situation of the PDMS already loaded with air before the liquid enters the channel and $P_{i}$ is the artificially controlled pressure imposed by the pressure controller. Equation (7) can be rewritten as

$$
\begin{aligned}
& \left(1-L_{f} / L\right)^{P_{0}} / P_{i} \\
& \quad=\exp \left\{\frac{1}{\psi}\left[\tau \exp \left(-\frac{1}{4 \tau}\right)-\frac{1}{4} \Gamma\left(0, \frac{1}{4 \tau}\right)\right]\right\},
\end{aligned}
$$

where $\psi=-t_{1}\left(P_{i} / P_{0}\right) / t_{0}$ and $\tau=t / t_{0}$.

Plotting $\left(1-L_{f} / L\right)^{P_{0} / P_{i}}$ against time $t$ in Fig. 3(b), all four data sets collapse and have a good agreement with the predictions from Eq. (8), which confirms the rationality of the model and the value of the fitting factor $\xi_{0}$.

With the above model, we then investigate the filling process of EGaIn in the microchannels. EGaIn is a low-toxicity electrically conductive liquid metal at room temperature. The eutectic alloy is composed of $75.5 \%$ $\mathrm{Ga}$ and $24.5 \%$ In by weight. The resistivity of EGaIn is 

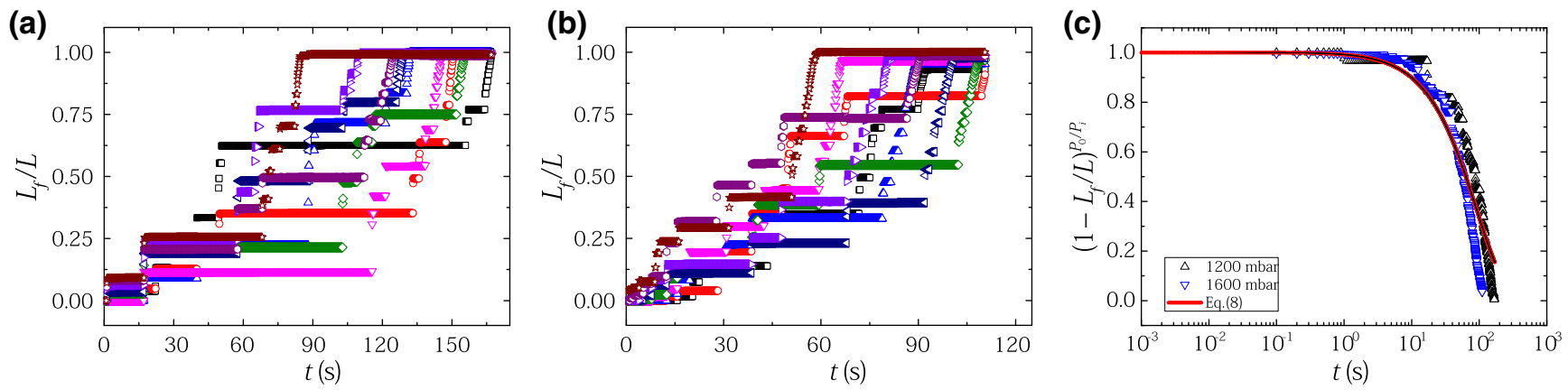

FIG. 4. (a) Experimental data of the nondimensional length of the EGaIn filled in each part of each channel as a function of time, under an imposed inlet pressure of (a) 1200 mbar and (b) 1600 mbar. Each color represents the filling process in each one of the nine microchannels considered. (c) Normalized filling length as a function of time for EGaIn. The open circles are the arithmetic average of the filling length of nine adjacent fingers at the same time and the red solid curves denote results based on the theoretical model.

$29.4 \times 10^{-6} \Omega \mathrm{cm}$, which is about eight orders of magnitude more conductive than tap water [33]. EGaIn is quickly gaining in popularity due to the vast number of applications in microfluidics such as pumps, valves, mixers, electrodes, sensors, and heaters [33]. We later use EGaIn-filled IDTs to demonstrate the generation of SAWs. Figure 4 shows both experimental and theoretical data for the case of EGaIn. The inlet pressures are 1200 and 1600 mbar. We choose these pressures for a number of reasons. At low pressures (1000 mbar or below), the filling time is relatively long (more than a few minutes) and the process is deemed inefficient for the fabrication of IDTs. At high pressures (above 1600 mbar), delamination may occur due to the relatively weak bond strength between PDMS and lithium niobate. The filling observations obtained at the above pressures are adequately sufficient for a qualitative comparison between both fluids (DI water and EGaIn). The obtained experimental results also provide ample data for theoretical modeling.

Compared with the filling process of DI water, intermittencies or stepwise filling can be observed for EGaIn. We attribute this interesting phenomenon to the formation of a thin oxide layer. This oxide layer forms instantaneously when EGaIn is exposed to air $[33,34]$. The filling process can be described as a continuous repetition of the following three steps: (1) filling, (2) formation of the oxidized layer, and (3) breaking the oxidized layer. This repetitive behavior is captured and depicted in both Figs. 4(a) and 4(b). We simplify the analysis by focusing on the average filling process in each single finger (IDT). We obtain the arithmetic average of the filling length of nine adjacent fingers and normalize the filling length as a function of time. The solid continuous curve in Fig. 4(c) shows the theoretical modeling using Eq. (8). The fitting parameter is $\xi_{0}=1.66$. Recall that $\xi_{0}$ represents the initial situation of the PDMS already loaded with air at atmospheric pressure $P_{0}$ before the liquid enters the channel. However, for the filling of liquid metal, the process is not only dominated by the initial condition of the PDMS channel prior to the liquid injection, but it is also influenced by the stochastic features during its filling process, as shown in Figs. 4(a) and 4(b). As a result, a different fitting parameter $\xi_{0}=1.66$, rather than $\xi_{0}=2.103$, is used in the model. We would like to emphasize that our current model does not agree flawlessly with the observed experimental data as that of water. Slight deviations from the theoretical model can be observed at various filling stages. Nevertheless, the model can still be considered as reasonable for both imposed inlet pressures of 1200 and 1600 mbar. The actual mechanism of the formation and breaking of the oxide layer is very complex and a more detailed analysis is currently beyond the scope of this paper. This complex mechanism is studied in detail in a separate work [35].

Finally, we demonstrate the generation of SAWs using an open-space droplet. The IDTs are filled with EGaIn using the proposed pressurized mechanism. This
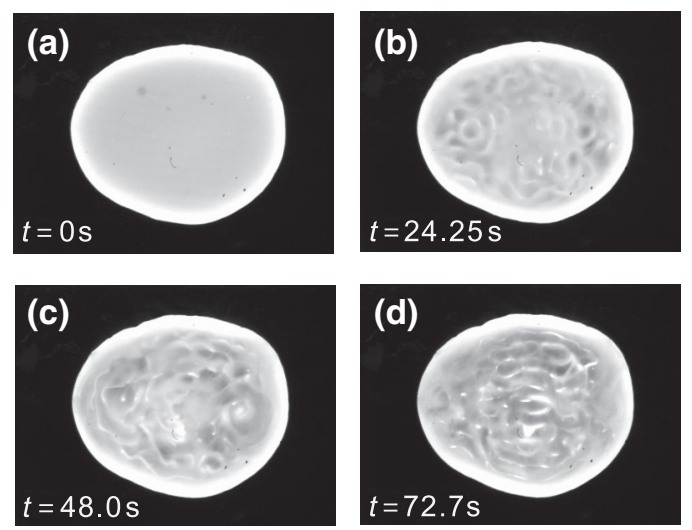

FIG. 5. Image sequence showing different flow patterns when the IDTs are activated. The frequency of the input signal is 4.161 MHz. The applied voltage is $200 \mathrm{mV}_{\mathrm{pp}}$. Acoustic streaming induced by traveling surface acoustic waves can be clearly observed (see Appendix D). 
demonstration clearly ascertains both the feasibility and applicability of the proposed concept. Here, we use 20 finger pairs and two separate microchannels are filled separately. The integrated PDMS microchannel and lithium niobate substrate is fabricated, bonded, and tested as reported in our previous work [17]. We characterize the $S_{11}$ measurement of the system using the network analyzer (Keysight technologies, 8753C) (see Appendix A).

To observe the surface acoustic waves generated by the IDT channels, a $0.5-\mu 1$ droplet loaded with $0.02 \%$ volume ratio of fluorescent particles (PSF001UM, Magsphere) is pipetted and placed about $7 \mathrm{~mm}$ away from the IDTs. We then apply an alternating current (ac) voltage of about $200 \mathrm{mV}_{\mathrm{pp}}$ at a frequency of $4.161 \mathrm{MHz}$. Figure 5 shows image frames of the droplet at different time squences. The images clearly show flow patterns induced by a streaming vortex. These flow patterns are similar to the one previously reported [36-39].

\section{CONCLUSION}

In conclusion, the pressurized filling processes of both DI water and EGaIn in a porous closed-end microchannel are experimentally and theoretically studied. For the case of DI water, our proposed model agrees relatively well with the experimental data. In the case of EGaIn, the theoretical model and the experimental data fit reasonably with slight deviations. We also demonstrate that the proposed filled IDTs can be used to generate SAWs to induce streaming in an open droplet. We envisage that the self-aligned comb-shaped single-electrode IDTs will be adopted in the microfluidic community due to their simplicity.

\section{ACKNOWLEDGMENTS}

This work is performed at the Queensland Node of the Australian National Fabrication Facility, a company established under the National Collaborative Research Infrastructure Strategy to provide nano- and microfabrication facilities for Australia's researchers. S.H.T. and N.T.N. gratefully acknowledge the support of the Australian Research Council (ARC) Discovery Early Career Researcher Award (DECRA) (Grant No. DE170100600), and Griffith University-Simon Fraser University Collaboration grant. W.G. and H.D.X. are grateful for the support by the National Natural Science Foundation of China (Grants No. 11472094, No. 11772259, and No. U1613227), and the 111 project of China (Grant No. B17037).

\section{APPENDIX A: RESONANCE FREQUENCIES OF THE DEVICE}

A network analyzer with a working bandwidth ranging from 0 to $30 \mathrm{MHz}$ is used to characterize the resonance frequencies of the fabricated device. The device is measured

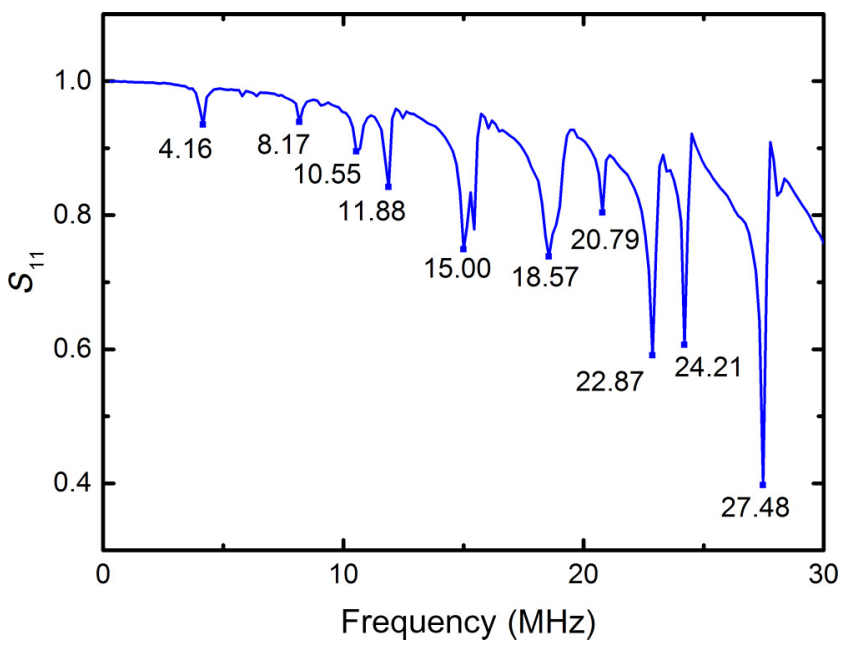

FIG. 6. Measured response frequencies obtained from one-port measurement $\left(S_{11}\right)$ of the network analyzer using an IDT device with both fingers filled with EGaIn.

using one port $\left(S_{11}\right)$. One-port measurement requires only one IDT as the emitter and receiver. In this method, an ac signal with fixed input power but varying frequencies is applied to the IDT. The reflected signal, which travels back from the device along the same signal cable, is used to transmit the input signal. The resonant frequency is determined when the output has a minimum value.

Figure 6 shows the measured signals $\left(S_{11}\right)$ when the interdigital channels are filled with EGaIn. Here, we choose the first-order frequency $(4.161 \mathrm{MHz})$ as the frequency of the input signal. The measured first resonance frequency agrees well with the theoretical resonance frequency of $4.975 \mathrm{MHz}(f=c / \lambda, c$ is about $3980 \mathrm{~m} / \mathrm{s}$, and $\lambda$ is $800 \mu \mathrm{m})$.

\section{APPENDIX B: VIDEO OF WATER FILLING}

This video compilation shows the effects of liquid filling using multiple inlet pressures. Each separate video is captured at a low frame rate of $10 \mathrm{frames} / \mathrm{s}$ and played at a rate of $125 \mathrm{frames} / \mathrm{s}$ [40]. For proper segregation of each liquid column in the different videos, the dye concentrations used at each pressure are slightly adjusted without affecting the fluidic properties of the DI water used.

\section{APPENDIX C: VIDEO OF LIQUID METAL FILLING}

This video shows the filling of the liquid metal (EGaIn, Sigma Aldrich) with inlet pressure at $1600 \mathrm{mBar}$ [40]. The video captures the stepped motion of the liquid metal. This motion is attributed to the constant formation and breaking of the oxide layer formed with exposure to air. This video is captured at 10 frames/s and played at a rate of 50 frames/s. 


\section{APPENDIX D: VIDEO OF SAW-INDUCED DROPLET STREAMING}

This video shows droplet streaming induced by a traveling surface acoustic wave [40]. The video captures the SAW-actuated particle motion inside a droplet. This video is captured at 20 frames/s and played at a rate of 20 frames/s.

[1] Z. Wang and J. Zhe, Recent advances in particle and droplet manipulation for lab-on-a-chip devices based on surface acoustic waves, Lab Chip 11, 1280 (2011).

[2] G. Destgeer and H. J. Sung, Recent advances in microfluidic actuation and micro-object manipulation via surface acoustic waves, Lab Chip 15, 2722 (2015).

[3] L. Y. Yeo and J. R. Friend, Surface acoustic wave microfluidics, Annu. Rev. Fluid Mech. 46, 379 (2014).

[4] Y. Xie, N. Nama, P. Li, Z. Mao, P.-H. Huang, C. Zhao, F. Costanzo, and T. J. Huang, Probing cell deformability via acoustically actuated bubbles, Small 12, 902 (2016).

[5] A. Ozcelik, N. Nama, P.-H. Huang, M. Kaynak, M. R. McReynolds, W. Hanna-Rose, and T. J. Huang, Acoustofluidic rotational manipulation of cells and organisms using oscillating solid structures, Small 12, 5120 (2016).

[6] L. Y. Yeo, H.-C. Chang, P. P. Chan, and J. R. Friend, Microfluidic devices for bioapplications, Small 7, 12 (2011).

[7] http://pubs.rsc.org/en/journals/articlecollectionlanding?ser code $=$ lc\&themeid $=522$ ee $1 \mathrm{~d} 1-9 \mathrm{ee} 9-4 \mathrm{~b} 13$-aed6-77c4d8fd99 44.

[8] R. M. White and F. W. Voltmer, Direct piezoelectric coupling to surface elastic waves, Appl. Phys. Lett. 7, 314 (1965).

[9] D. Morgan, Surface Acoustic Wave Filters (Elsevier, Amsterdam, 2007).

[10] Z. Z. Chong, S. H. Tan, A. M. Ganan-Calvo, S. B. Tor, N. H. Loh, and N.-T. Nguyen, Active droplet generation in micro fluidics, Lab Chip 16, 35 (2016).

[11] Z. Z. Chong, S. B. Tor, N. H. Loh, T. N. Wong, A. M. Ganan-Calvo, S. H. Tan, and N.-T. Nguyen, Acoustofluidic control of bubble size in microfluidic flow-focusing configuration, Lab Chip 15, 996 (2015).

[12] M. Sesen, T. Alan, and A. Neild, Microfluidic on-demand droplet merging using surface acoustic waves, Lab Chip 14, 3325 (2014).

[13] H.-D. Xi, H. Zheng, W. Guo, A. M. Ganan-Calvo, Y. Ai, C.-W. Tsao, J. Zhou, W. Li, Y. Huang, and N.-T. Nguyen, Active droplet sorting in microfluidics: A review, Lab Chip 17, 751 (2017).

[14] X. Ding, P. Li, S.-C. S. Lin, Z. S. Stratton, N. Nama, F. Guo, D. Slotcavage, X. Mao, J. Shi, F. Costanzo, and T. J. Huang, Surface acoustic wave microfluidics, Lab Chip 13, 3626 (2013).

[15] J. Min and A. J. Baeumner, Characterization and optimization of interdigitated ultramicroelectrode arrays as electrochemical biosensor transducers, Electroanalysis 16, 724 (2004).
[16] T. Franke, A. R. Abate, D. A. Weitz, and A. Wixforth, Surface acoustic wave (saw) directed droplet flow in microfluidics for pdms devices, Lab Chip 9, 2625 (2009).

[17] Z. Ma, A. J. Teo, S. H. Tan, Y. Ai, and N.-T. Nguyen, Self-aligned interdigitated transducers for acoustofluidics, Micromachines 7, 216 (2016).

[18] A. Siegel, D. Bruzewicz, D. Weibel, and G. Whitesides, Microsolidics: Fabrication of three-dimensional metallic microstructures in poly(dimethylsiloxane), Adv. Mater. 19, 727 (2007).

[19] S. H. Tan, F. Maes, B. Semin, J. Vrignon, and J.-C. Baret, The microfluidic jukebox, Sci. Rep. 4, 4787 (2014).

[20] H.-D. Xi, W. Guo, M. Leniart, Z. Z. Chong, and S. H. Tan, Ac electric field induced droplet deformation in a microfluidic t-junction, Lab Chip 16, 2982 (2016).

[21] J. Nam and C. S. Lim, A conductive liquid-based surface acoustic wave device, Lab Chip 16, 3750 (2016).

[22] J. Nam, J. Y. Kim, and C. S. Lim, Continuous sheathless microparticle and cell patterning using cl-ssaws (conductive liquid-based standing surface acoustic waves), AIP Adv. 7, 015314 (2017).

[23] T.-T. Wu and I.-H. Chang, Actuating and detecting of microdroplet using slanted finger interdigital transducers, J. Appl. Phys. 98, 024903 (2005).

[24] M. K. Tan, J. R. Friend, and L. Y. Yeo, Direct visualization of surface acoustic waves along substrates using smoke particles, Appl. Phys. Lett. 91, 224101 (2007).

[25] X. Ding, S.-C. S. Lin, M. I. Lapsley, S. Li, X. Guo, C. Y. Chan, I.-K. Chiang, L. Wang, J. P. McCoy, and T. J. Huang, Standing surface acoustic wave (ssaw) based multichannel cell sorting, Lab Chip 12, 4228 (2012).

[26] L. Schmid, D. A. Weitz, and T. Franke, Sorting drops and cells with acoustics: Acoustic microfluidic fluorescenceactivated cell sorter, Lab Chip 14, 3710 (2014).

[27] V. Skowronek, R. W. Rambach, L. Schmid, K. Haase, and T. Franke, Particle deflection in a poly(dimethylsiloxane) microchannel using a propagating surface acoustic wave: Size and frequency dependence, Anal. Chem. 85, 9955 (2013).

[28] S. H. Tan, N.-T. Nguyen, Y. C. Chua, and T. G. Kang, Oxygen plasma treatment for reducing hydrophobicity of a sealed polydimethylsiloxane microchannel, Biomicrofluidics 4, 032204 (2010).

[29] A. J. Teo, K.-H. H. Li, N.-T. Nguyen, W. Guo, N. Heere, H.D. Xi, C.-W. Tsao, W. Li, and S. H. Tan, Negative pressure induced droplet generation in a microfluidic flow-focusing device, Anal. Chem. 89, 4387 (2017).

[30] V. N. Phan, N.-T. Nguyen, C. Yang, P. Joseph, L. Djeghlaf, D. Bourrier, and A.-M. Gue, Capillary filling in closed end nanochannels, Langmuir 26, 13251 (2010).

[31] G. C. Randall and P. S. Doyle, Permeation-driven flow in poly(dimethylsiloxane) microfluidic devices, Proc. Natl. Acad. Sci. 102, 10813 (2005).

[32] M. Sadrzadeh, K. Shahidi, and T. Mohammadi, Synthesis and gas permeation properties of a single layer pdms membrane, J. Appl. Polym. Sci. 117, 33 (2010).

[33] K. Khoshmanesh, S.-Y. Tang, J. Y. Zhu, S. Schaefer, A. Mitchell, K. Kalantar-zadeh, and M. D. Dickey, Liquid metal enabled microfluidics, Lab Chip 17, 974 (2017). 
[34] T. D. Martz, D. Bardin, P. S. Sheeran, A. P. Lee, and P. A. Dayton, Microfluidic generation of acoustically active nanodroplets, Small 8, 1876 (2012).

[35] A. M. Gañán-Calvo, W. Guo, H.-D. Xi, A. J. Teo, N.-T. Nguyen, and S. H. Tan, Pressure-driven filling of liquid metal inclosed-end microchannels, Phys. Rev. E 98, 032602 (2018).

[36] H. Li, J. R. Friend, and L. Y. Yeo, Microfluidic Colloidal Island Formation and Erasure Induced by Surface Acoustic Wave Radiation, Phys. Rev. Lett. 101, 084502 (2008).

[37] M. Wiklund, R. Green, and M. Ohlin, Acoustofluidics 14: Applications of acoustic streaming in microfluidic devices, Lab Chip 12, 2438 (2012).
[38] M. Alghane, B. X. Chen, Y. Q. Fu, Y. Li, J. K. Luo, and A. J. Walton, Experimental and numerical investigation of acoustic streaming excited by using a surface acoustic wave device on a $128 \mathrm{yx}-$ linbo 3 substrate, J. Micromech. Microeng. 21, 015005 (2011).

[39] R. J. Shilton, M. Travagliati, F. Beltram, and M. Cecchini, Nanoliter-droplet acoustic streaming via ultra high frequency surface acoustic waves, Adv. Mater. 26, 4941 (2014).

[40] See Supplemental Material at http://link.aps.org/supple mental/10.1103/PhysRevApplied.10.054045 for video of water filling. 